\title{
Requirements for EV Charge Stations with Photovoltaic Generation and Storage
}

\author{
J.-I. Cairo, Member IEEE, Andreas Sumper, Senior Member IEEE.
}

\begin{abstract}
With the increase in electric mobility, public and private charge facilities will be required to provide solutions adjusted to the actual needs and requirements. A new methodology for dimensioning charge stations for electric vehicles (EVs) is presented in this paper. Such proposal is based on the Markov chains, and uses as output rates, the capabilities of the charge station in providing energy from the sun through photovoltaic generation, stored energy in local batteries and also as a back-up the power grid. A numerical result is also presented for a better understanding of the process.
\end{abstract}

Index Terms-Battery, Death chain, electric vehicles, Markov, Poisson, power converter, photovoltaic, probability, queuing, renewable, Request of charge (RoC).

\section{INTRODUCTION}

$\mathrm{T}_{\mathrm{g}}^{\mathrm{H}}$ HE introduction of electric vehicles (EVs) is slowly growing as the availability of electric cars is increasing, the prices are decreasing and the infrastructure for charging EVs is improving. It has been reported [1] the big reductions in greenhouse gas emissions from EVs in comparison to conventional vehicles. Since the transport industry represents about $24 \%$ of the global green house emissions [2], companies and governments are increasing the efforts in developing EV and facilitating its introduction.

A growing world population and an increasing standard of living, are major global trends that pose a number of problems in the upcoming years. One of the challenges resides on how to satisfy growing demand for energy, which by the year 2030 is expected to increase by $44 \%$ compared to the level of 2010 [3]. In addition to the use of electricity for mobility and transport in order to reduce pollution and contribute to green house emissions, the use of renewable energies, are also contributing to such trend, in that sense, smart microgrids fueled by renewable energy resources are becoming a popular green approach [4], and will contribute as well to the introduction of EVs. There is then a trend towards helping the introduction of renewable energies when using EVs, they might need such resource to optimize energy efficiency and help in energy resources. However, renewable energies which

This research was supported by the EIT and KIC-Innoenergy under the Project ACTIVE SUBSTATIONS (KIC-ASS).

Josep Ignasi Cairo is working at the Research Institute for Energy of Catalonia (IREC), located in Barcelona (Spain), at the department of Electricity and Power Electronics, e-mail: icairo@irec.cat.

Andreas Sumper is working at Dept. of Electric Engineering at CITCEA (UPC) ESEIB and at IREC in Barcelona (Spain) at the dept. of Electricity and Power Electronics, e-mail: andreas.sumper@upc.edu. are highly intermittent in nature [5], have to deal with EVs which arrive at the microgrid randomly. Therefore, the stochastic nature of both renewable energy sources and EVs should be carefully considered.

In addition of having renewable energy helping in the charging of EVs, uncontrolled daytime EV charging pattern may reduce the optimization of, for example solar energy use for charging purposes, and increase stress on the power system during the peak time, which may result in grid outages [6] due to limitations in distribution transformers. Here is a contribution of this paper, the help of refilling management together with the use of renewable energy, mainly photovoltaic energy, by suggesting methods for anticipating temporal EV charging demand, based on queuing theory.

Even with the inherent difficulty of photovoltaic power that introduces a specific complexity because of the variation of this resource availability over the planning zone, the charging management will optimize always the use of solar power when available. In the case of residential charging facilities, it is also of interest to consider the possibility of using the EVs, together with other price-elastic residential loads such as washing machines, that with the provision of communication devices, should be able to communicate with the smart meter of the house, which is in contact with the utility and be used to make a proper energy management system. That brings another level of complexity to the system that will not be contemplated in this paper.

\section{ELECTRIC MobILITY NEEDS}

There are several classes of EVs in the system, each class with different charging capacity and quality of service requirements [12] (e.g., emergency vehicles will require higher service availability). This study has been carried out based on actual data from a city of Spain (Vitoria). Based on this data it has been proposed some hypothesis and assumptions that focuses the scope of possibilities without eliminating the thoroughness of the methodology

\section{A. Observed area}

Data for the case scenario used in this paper, has been obtained from a $13.2 \mathrm{kV}$ network in the Spanish electric system, in the city of Vitoria (Álava) powered by two power transformers. The conventional demand in that precise region is of $36 \mathrm{MW}$ spread in different areas as described in table I.

The assumptions for the EV penetration are taken from a study in the same project context, with the aim of estimating the power requested for charging EV's in the 2010-2020 
horizon. From the different scenarios here described, for this study it has just been considered the case of residential areas.

\section{B. Assumptions on mobility}

The other source of information provided for this study comes from the project MOVILIA2006 [10], which considers an average consumption for the EV of $200 \mathrm{Wh} / \mathrm{km}$, a mean traveling distance of $15 \mathrm{~km}$, and the average speed for the electric vehicles is supposed to be $30 \mathrm{~km} / \mathrm{h}$, mainly inside urban areas. The capacity of the battery will depend on the type of vehicle, for this work it is supposed an average BEV with $25 \mathrm{kWh}$ of capacity. From these considerations the daily request of charge (RoC) will be in the order of $1 / 8$ of the battery capacity; $R o C=200 \mathrm{Wh} / \mathrm{km} \cdot 15 \mathrm{~km}=3 \mathrm{kWh}$.

From the work [10], it is also obtained the number of commutes in auto and motorcycle either in a mean labor day, as well as non-labor day in the province of Alava.

- Number of Commutes in average labor day: 41151

- Number of Commutes in average non-labor day: 38022

There is also information related to the number of commutes realized by the different type of EVs, and it is according to the following distribution:

- $\quad$ PHEV: $36.83 \%$

- BEV: $45.02 \%$

- Electric motorcycle: $18.15 \%$

In figure 1 one can see the distribution during a whole day (24 hours) of the number of commutes due to people returning home in a labor day [10].

TABLE I

DISTRIBUTION OF ENERGY DEMANDED IN THE METROPOLITAN AREA OF ALAVA (SPAIN) BY SECTORS

\begin{tabular}{lllll}
\hline \hline $\begin{array}{l}\text { Type of } \\
\text { Area }\end{array}$ & Residential & Commercial & Industrial & Others \\
\hline Load (\%) & $13 \%$ & $7 \%$ & $68.4 \%$ & $11.6 \%$ \\
\hline
\end{tabular}

As a premise, it has been chosen for this study, the time of the day during which the users will most probably use for recharging their EV's. That time corresponds during evening night hours (from $16 \mathrm{pm}$ to $8 \mathrm{am}$ ), and this premise is used for the next part of the study.

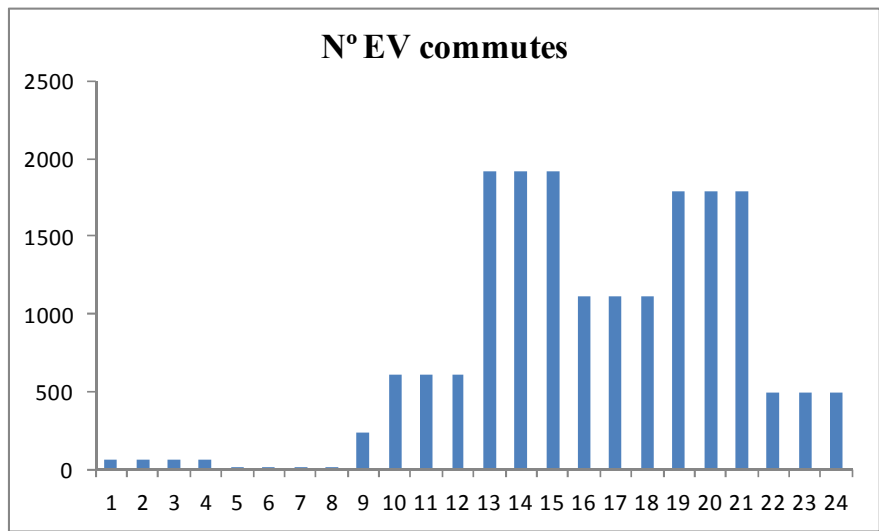

Fig. 1 Number of commutes arriving at the user home during a whole labor day.

\section{Charging RESOURCES}

To solve the problem proposed, it is needed to formally propose the necessities and the capabilities in order to provide with a feasible solution for the refilling or charging needs. In that sense the problem becomes defined by using several scenarios and use cases in relation to charging capabilities of a facility including not direct connection into the main power grid, but also the use of solar energy generated by photovoltaic panels, or storage energy, coming from a set of battery packs that are being used as an additional resource of energy. The EV that will be connected in such resources, will use at first instance the maximum of the photovoltaic power, this is a practice already being used [13]. Also other sources of renewable energy could be used for this purpose. In case there is not enough energy coming from solar panels, the energy from the battery pack can be used to be injected into the battery of the electric vehicle, so a direct transfer of energy between batteries will be done. The last resource to be used is the power connection into the main grid. Based on price signals (selling price from utility or energy retailer), the management system that controls such energy activity, will switch from one or another energy source according to the best cost performance, giving priority always the use of renewable energy sources. So a compromise between economical, technical and $\mathrm{CO}_{2}$ emissions are present.

\section{A. Power Grid supply}

From the power grid, through the distribution transformer, available power will be provided for charging the batteries of the EVs. Power from the grid will be always an available resource and is the one that should be minimized, giving priority to other sources of energy such as renewable when available. So this energy resource is balancing the other sources in order to provide a zero net balance between demand and generation.

\section{B. Photovoltaic and grid power supply}

This source of energy will be intermittent during day time and should always be used for the purposes of charging the batteries, reducing hence, the need for using power from the grid. In case no electric vehicle is connected, instead of charging the batteries of the EV, it will be used to charge the battery pack, that it is available in this use case. Only in the case there is no need to charge EV, and the local battery pack is full, one can think of injecting the energy from solar panels back into the main power grid. This is the most common use in facilities with no storage capacity.

\section{Battery storage}

In the case presented here, in order to broaden the possibilities under study, it is also considered a local storage system provided by battery packs. Such option becomes very interesting in facilities such as the ones investigated, where a source of renewable energy is a photovoltaic system [7], in this sense, the storage unit is balancing the intermittent source of energy coming from the sun. This local storage unit can be charged from solar panels, so the photovoltaic energy is also used in case there is no load (no EVs). Other possibility is to 
use this energy to charge the EV for example when energy prices are high, or when the energy needs exceeds the capacities of the network. Load shading is one of the important uses of battery storage energy.

\section{CASE STUDY}

In order to provide the maximum inside into the problem, and contemplate a wide scenario also in terms el EV charging requirements, it will be considered two types of EVs charging needs $R_{1}\left(\operatorname{RoC}_{1}\right)$ and $R_{2}\left(\operatorname{RoC}_{2}\right)$. Each one of them will generate RoC according to Poisson regimes with ratios $\lambda_{1}$ and $\lambda_{2}$. Both are generating some needs for charging that need to be supported by the infrastructure. From its nature, the energetic requirements of the RoC, presents an exponential distribution versus time and, the generation of the $\mathrm{RoC}$, are independent one from another.

The capacity of the network to provide energy into the EVs is assigned so that the use of photovoltaic energy is maximized versus the use of energy from the power grid. This is a resource that is dynamically assigned depending on the demand. In that sense it is possible to differentiate two zones of use figure 2 :

- A zone: If the number of EVs, is zero $N q=0$.

- B zone: If the number of EVs is lower than the ones at level1; $N q<L_{l}$.

- C zone: If the number of EVs is in between levell and level2; $L_{2}>N q>L_{1}$.

- D zone: If the number of EVs is higher than the ones at level $2 \mathrm{Nq} \geq L_{2}$.

Where $N q$ is the number of electric vehicles waiting or expecting to get charged from the network and, it is directly proportional to the RoC. $L_{l}$ is the number of EVs that are being charged based on solar energy, it corresponds to the threshold level of $\mathrm{RoC}$ (or $N q$ ) above which the photovoltaic energy cannot provide all the required energy to charge the EVs and it will be necessary to use energy from other sources such a battery or from the main grid. $L_{2}$ is the threshold level of EV charging request (higher than $L_{1}$ ) above which neither the photovoltaic panels nor the storage energy in the local battery cannot supply the $\mathrm{RoC}$, and it becomes necessary to use energy from the power grid.

There is a direct relationship between the expected number of EVs and the prediction of energy requirements for the network. EVs will incorporate communication means, so that the information related to the level of charge requested by the user will be sent to the local charging manager, local manager center or the management system responsible to assign the appropriate power source into the system. The communication channel is not only used to coordinate the charge level requirements, but also to sent information related to the user identification, data from the EV or battery data such as type of battery, possibility to use Vehicle two the Grid (V2G), or connector type. This can be done remotely via wireless communications or once the EV is plugged (conductive charging) or located at the proximities of the charging post (inductive charging) via wired line, or RFID - NFC means.
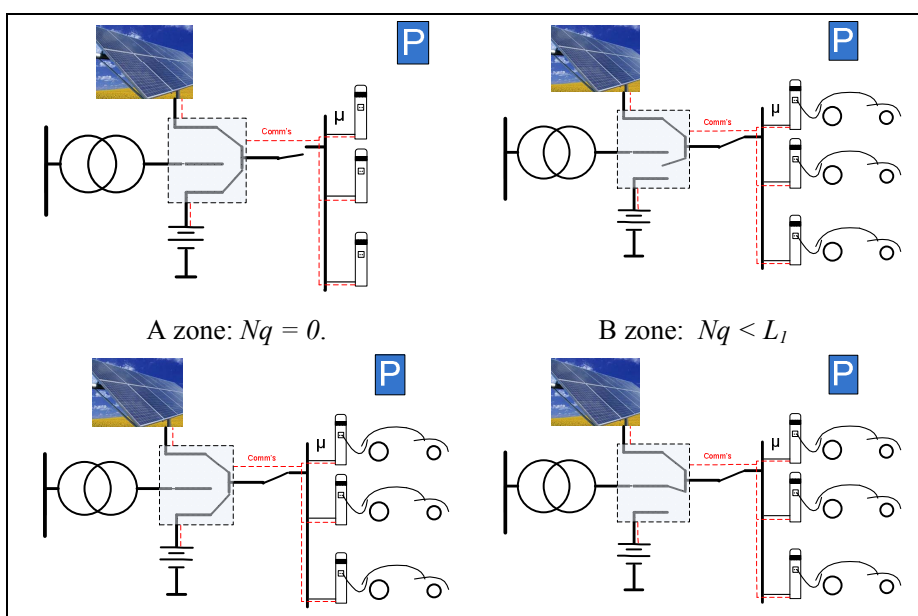

C zone: $L_{2}>N q>L_{l}$.

D zone: $N q \geq L_{2}$.

Fig. 2 Diagram of the different configurations for the charging spots

\section{A. Average arrival rate}

As mentioned before, there are different needs for EV charging requests, charging profiles and also arrival rates. There are different scenarios for charging stations that may also appear such as street areas, public parking's, domestic charging or in private parking's from a community or private institution. In any of these cases, one of the most employed statistics to model the arrivals of EVs, so to estimate the RoC level, due to its simplicity and properties is the Poisson process.

With previous hypothesis, the probability of having $n$ arrivals of EVs at time $T$ considering arrival rate $\lambda$ follows:

$$
P_{n}(t)=\frac{(\lambda T)^{n}}{n !} e^{-\lambda T}
$$

Figure 1 represents data from the number of commutes in the scenario presented before and, in the case of this study a time window of 16 hours from $16 \mathrm{pm}$ until 8 am is considered. In that window, we can obtain a distribution rate of $\lambda=4.2$, with $f_{i}$ as the relative frequency of observations:

$$
\lambda=\bar{X}=\frac{1}{n} \sum_{i=0}^{k} x_{i} \cdot f_{i} \quad f_{1}+f_{2}+\ldots+f_{k}=n
$$

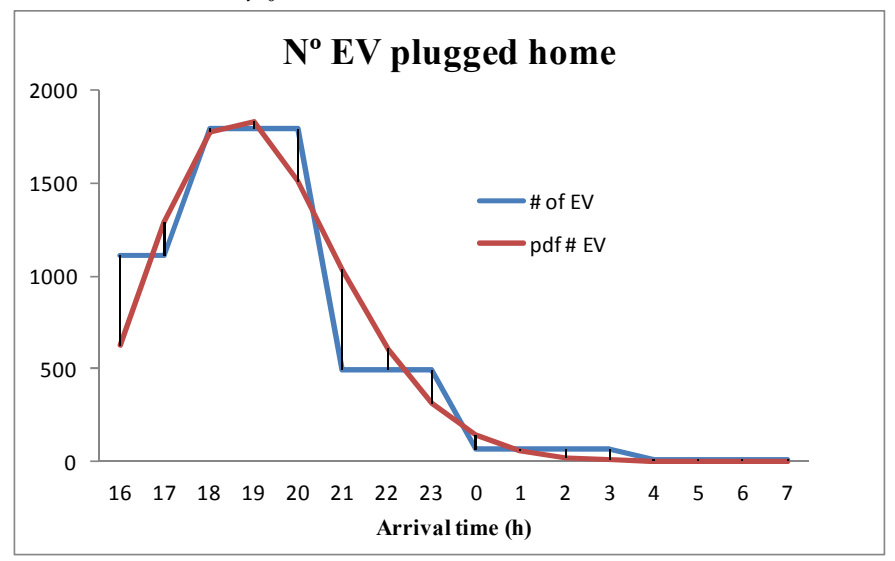

Fig. 3 Poisson distribution of the number of home arrivals from $16 \mathrm{~h}$ until 7h.in a labor day. 
In figure 3 it is plotted the Poisson distribution of arrival probabilities comparing the data from the study [2] and the estimated data by Poisson law.

As a premise one can consider two types of EVs, with different needs regarding charging batteries, so it may be estimated the following:

- $\quad N^{e v}{ }_{1}$ number of EVs are arriving at a rate $\lambda_{I}$

- $\quad N^{e v}{ }_{2}$ number of EVs are arriving at a rate $\lambda_{2}$

Arrival rate of EVs, will be $\lambda=N^{e v}{ }_{1} \cdot \lambda_{1}+N^{e v}{ }_{2} \cdot \lambda_{2}$. In the case of this study, just one average arrival rate $\lambda$ of EVs is considered.

\section{B. Probability State}

It is of interested to find out the probabilities of the states or zones defined previously, in order to obtain an idea of the utilization of the different resources (solar panels, local storage, and power network). One option is to start defining the ratio $a=\lambda / \mu$, and finding the probability that there are EVs with a RoC level required lower than Levell $\left(L_{I}\right)$, such as zone B.

In order to proceed with such calculation, it will be used Markov process. Such process uses exponential probability distribution, corresponding to a system where more events are expected below the mean value of the random variables than above it, this probabilistic pattern is a classical model for electric systems reliability studies [8-9].

From the specification of zones firstly one specifies the state transient diagram that corresponds to the system of charging stations and some servicing rate of EVs. The process that best describes the situation presented is the birth and death process that is characterized in steady state by the following equation:

$$
\begin{cases}0=\mu_{n+1} \cdot P_{n+1}+\lambda_{n-1} \cdot P_{n-1}-\left(\lambda_{n}+\mu_{n}\right) P_{n} ; & n>0 \\ 0=\mu_{1} \cdot P_{1}-\lambda_{0} \cdot P_{0} ; & n=0\end{cases}
$$

Where $\mu$ is the stepping backwards distribution rate, also known as service rate [11]. In this case the arrival rate is constant and fixed to $\lambda_{i}=\lambda$ and the service rate $\mu_{i}=\mu$ for $i=$ $1,2, \ldots L_{1}$, and $\mu_{i}=2 \mu$ for $i=L_{1+1}, L_{1+2}, . . L_{2}$, and $\mu_{i}=3 \mu$ for $i$ $=L_{2}, L_{2+1}$,

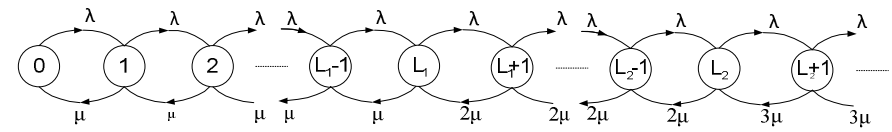

Fig. 4. Diagram of the Markov process

Solving the previous state diagram for zone B, starting at state 0 and arriving at state $L_{1}$, one can obtain:

$$
p_{k}=p_{0} \prod_{i=0}^{k-1} \frac{\lambda_{i}}{\mu_{i+1}}=p_{0}\left(\frac{\lambda}{\mu}\right)^{k}=p_{0} \cdot a^{k}
$$

where $p_{k}$ is the probability of being at sate $k$.
Solving for zone C, where $L_{2}>N q>L_{1}$ :

$p_{k}=p_{0} \prod_{i=0}^{k-1} \frac{\lambda_{i}}{\mu_{i+1}}=p_{0} \prod_{i=0}^{L_{1}-1} \frac{\lambda}{\mu} \prod_{i=L_{1}}^{k-1} \frac{\lambda}{2 \mu}=p_{0} \cdot 2^{L_{1}}\left(\frac{a}{2}\right)^{k}$

It is already interesting to note the exponential dependency of the probability of occupancy of one of the states and the length of the occupation $L_{1}$. Finally it is solved for the zone D, where $N q \geq L_{2}$ :

$$
\begin{aligned}
p_{k}=p_{0} \prod_{i=0}^{k-1} \frac{\lambda_{i}}{\mu_{i+1}} & =p_{0} \prod_{i=0}^{L_{1}-1} \frac{\lambda}{\mu} \prod_{i=L_{1}}^{k-1} \frac{\lambda}{2 \mu} \prod_{i=L_{2}}^{k-1} \frac{\lambda}{3 \mu} \\
& =p_{0} \cdot \frac{3^{L_{2}}}{2^{L_{2}-L_{1}}}\left(\frac{a}{3}\right)^{k}
\end{aligned}
$$

It is necessary to use previous expressions to obtain the value of the probability of being at the state where there are no EVs charging at any of the available charging points. This probability corresponds after operating to:

$$
p_{0}=\left[1+\sum_{k=1}^{\infty} \prod_{i=0}^{k-1} \frac{\lambda}{\mu_{i+1}}\right]^{-1}
$$

and solving the expression further one can obtain:

$$
\begin{aligned}
p_{0}=\left[\frac{1-a^{L_{1}+1}}{1-a}\right. & +2^{L_{1}} \frac{\left(\frac{a}{2}\right)^{L_{1}+1}-\left(\frac{a}{2}\right)^{L_{2}+1}}{1-(a / 2)} \\
& \left.+\frac{3^{L_{2}}}{2^{L_{2}-L_{1}}} \frac{\left(\frac{a}{3}\right)^{L_{2}+1}}{1-(a / 3)}\right]^{-1}
\end{aligned}
$$

\section{NUMERICAL EXAMPLE}

For a better understanding of the methodology, it will be presented a numerical example, with values that are coming from a real situation presented early in the paper. For simplicity, it is assumed that photovoltaic and battery capacity are rated with the same power capacity $(4.7 \mathrm{kWh})$ with a maximum number of EVs that can provide energy equal to two $\left(\mathrm{L}_{1}=2\right)$, and the value of $a=2$.

The variables that encompass the numerical example from this point beyond are the following:

- $S o C_{i n}$ : Initial State Of Charge $(\mathrm{kWh})$

- $d_{t r}$ : Travelled distance by the EV (km)

- $C_{b a t}:$ Capacity of the battery $(\mathrm{kWh})$

- $C_{\text {eff: }}$ Coefficient related to the total efficiency including driving conditions based on driving patterns, traffic conditions, driver and electric engine efficiency (\%)

- $\quad E^{p v}$ : Energy taken from photovoltaic panel (kWh)

- $E^{S}$ : Energy taken from storage device $(\mathrm{kWh})$

- $E^{p c}$ : Energy taken from power converter (kWh) 
- $\quad N^{c p}$ : Number of charging posts

- $\quad N^{e v}$ : Number of electric vehicles.

When dimensioning charging requirements for the power grid network, one very well established requirement will be the need to full charge the EV, in the cases where the user arrives at its residence, and plug the EV during the afternoonevening hours. In that case the condition taken for granted will be to consider, that the battery is fully charged $(100 \%)$ at the departure time at first hour of the morning:

$$
S o C_{i n}=100-\frac{d_{t r} / 2}{C_{b a t} \cdot C_{e f f}} \cdot 100
$$

So the requested charge at the end of the day, just after commuting home will be $\mathrm{RoCi}=100-\mathrm{SoC}_{i n}$. The state of the battery after a whole day of commuting, will depend very much on the conditions of the traffic as well as the type of driver. In that sense different driving models patterns may exist, and could be considered by the analysis as stated in the definition of the variables above.

In this example, an average value also has been considered the arrival rate of EV at the users home, considering the total number of arrivals in Alava city, an estimating an average number of parking locations (residential parking's). At this point, there is no information on how the parking location are placed, a variable considered in this study is the number of such location in order to consider the average input rate.

\section{A. Computation of system probabilities}

With the values defined above and using (7) one can solve it to obtain $\mathrm{p}_{0}=3 \%$. From this value the probabilities that the EVs are being recharged by solar energy (either directly from photovoltaic panels and storage energy) is $\mathrm{p}_{\mathrm{B}}=48 \%$, and the probability of needing energy from the grid is $\mathrm{p}_{\mathrm{D}}=51 \%$.

\section{B. Number of EV's in the system}

Considering previous information, another parameter of interest for the local manager of the charging stations, will be the average value of EVs in the charging stations. That number would include the EVs being connected, and also, if any, the number of EVs awaiting to be charged. For that computation, it is required to add all the possibilities of the system with their local probabilities:

$$
\begin{aligned}
N=\sum_{k=0}^{\infty} k \cdot p_{k}= & \sum_{k=0}^{L} k \cdot a^{k} \cdot p_{0} \\
& +\sum_{k=L+1}^{\infty} k \cdot 3^{L}\left(\frac{a}{3}\right)^{k} \cdot p_{0}
\end{aligned}
$$

Doing some math into theses equation one can obtain the final relationship of:

$$
\begin{aligned}
& N \\
& =p_{0} \\
& \cdot a\left[\frac{(L+1) a^{L}(a-1)+1-a^{L+1}}{(1-a)^{2}}\right. \\
& \left.+3^{L} \frac{\frac{L+1}{3}\left(\frac{a}{3}\right)^{L}\left(1-\frac{a}{3}\right)+\frac{1}{3}\left(\frac{a}{3}\right)^{L+1}}{(1-a / 3)^{2}}\right]
\end{aligned}
$$

So in the case of this numerical example, the total number of EV's in the system is equal to $N^{e v}=3$. This number corresponds to the number of EVs that has been computed considering the probability of staying at state number zero $\left(p_{0}\right)$, the variable $a$, which depends on the arrival rate and service rate and, finally on the number of EVs that could be charged by either the photovoltaic panels capacity installed, as well as by the storage system.

\section{Remaining time in the charging stations}

It is also of interest, to compute the time that EVs will remain in charging locations to complete a full charge cycle. This value is very much related with the effectiveness of the system installed. In case the power network would be saturated, and could not provide enough energy, or the number of electrical recharging posts would not be sufficient, that number would be affected.

For this case example, as stated in table II, only one charging post or charging plug is considered in each residential area $\left(N^{c p}=1\right)$.

With the premises used in this example, it is possible to obtain the rate of service that up to the level $L_{l}$ correspond the parameter $\mu$. In this case, based that parameter $\mathrm{a}=2$. Then the service rate has a value of $\mu=3.4$, that corresponds a servicing rate once in the level $2\left(L_{2}\right)$ of roughly $3 \mu \approx 10$.

In addition it is also of interest to obtain the average time $(T)$ for an EV remaining plugged charging the battery by using the following expression:

$$
T=\frac{N^{e v}}{\lambda}
$$

Using the values of table II the average time one can get becomes equal to 43 minutes. This number may become of importance since will provide information of the usage or exploitation time of the charging facilities and, the number of such facilities that may be additionally required in case of saturation, or in case of having a long waiting time for the users.

TABLE II

COMPLEMENTARY DATA TO EVALUATE THE PROPOSED EXAMPLE

\begin{tabular}{ccccccc}
\hline \hline $\begin{array}{c}\mathrm{E}^{\mathrm{pc}} \\
(\mathrm{kWh})\end{array}$ & $\begin{array}{c}\mathrm{E}^{\mathrm{pv}} \\
(\mathrm{kWh})\end{array}$ & $\begin{array}{c}\mathrm{E}^{\mathrm{S}} \\
(\mathrm{kWh})\end{array}$ & $\begin{array}{c}\mathrm{a} \\
(\lambda / \mu)\end{array}$ & $\begin{array}{c}\mathrm{RoC} \\
(\mathrm{kWh})\end{array}$ & $\mathrm{N}^{\mathrm{ev}}$ & $\mathrm{N}^{\mathrm{cp}}$ \\
\hline 3.7 & 4.7 & 4.7 & 2 & 3 & 3 & 1 \\
\hline
\end{tabular}




\section{CONCLUSION}

In this paper has been presented a methodology for estimating the requirements in terms of charging needs in accordance to some prediction of charging necessities. What this study introduces, in difference to other studies is the use of probabilistic Markov chains to define the analysis, and also the introduction of several components into the study that broadens the applicability of the method.

Among the possibilities that exhibit such method are the use of photovoltaic generation as an additional parameter, or the possibility to include also local static battery storage with a certain capacity by means of battery packs. Such possibilities provide added value into the analysis since it includes renewable capacity to the charging system. The use of storage capacity with photovoltaic's generation optimizes the total capacity of the photovoltaic panels.

The method used based on death and birth chains, can estimate the probability of congestion of such resources, so it can forecast, based on basic assumptions of demand, several quantities. Some of these quantities are the exploitation of the resources available, what would be additional requirements in case of necessary, how the exploitation from distributed energy resources can be incorporated into the analysis, and the expected congestion of the system in terms of, number of EVs using the charging facilities or the time that will be using such facilities before being full charged by the system.

\section{REFERENCES}

[1] K. Parks, P. Denholm, and T. Markel, "Costs and emissions associated with plug-in hybrid electric vehicle charging in the Xcel Energy Colorado service territory," Technical Report NREL/TP-640-41410, May 2007.

[2] S. Labatt and R. R. White, Carbon Finance: The Financial Implications of Climate Change, John Wiley \& Son, Inc., 2007

[3] U.S. Energy Information Administration, "EIA - 2010 international energy outlook", EIA, Tech. Rep. DOE/EIA-0484(2010), May 2010.

[4] M. Shahidehpour, "Role of smart microgrid in a perfect power system", in Proc. 2010 IEEE Power and Energy Society General Meeting.

[5] S. Bu, F. R. Yu, and P. Liu, "Stochastic unit commitment in smart grid communications", in Proc.2011 IEEE Infocom11 Workshop on Green Communications and Networking.

[6] P. Mohseni and R. G. Stevie, "Electric vehicles: Holy grail or fool's gold", in Proc.2009 IEEE PES Gen. Meet., pp. 1 - 5.

[7] K. Yoshimi, M. Osawa, D. Yamashita, T. Niimura, R. Yokoyama, T. Masuda, H. Kondo, and T. Hirota, "Practical storage and utilization of household photovoltaic energy by electric vehicle battery", in IEEE PES Innovative Smart Grid Technologies Conf., 2012, pp. $1-8$.

[8] L.E. Aparicio, "Stochastic reliability study of the western 765, 400 and $230 \mathrm{kV}$ transmission lines maintained by EDELCA", in IEEE PES Transmission and Distribution conference and Exposition: Latin America, 2008, pp. 1 - 7.

[9] R. Billinton and K. E. Bollinger, "Transmission system reliability evaluation using Markiv processes", in IEEE Trans. PAS-87, No.2 Feb. 1968, pp. $538-547$.

[10] Project MOVILIA2006. Ministerio de Fomento

[11] Leonard Kleinrock, Queueing Systems: Volume I: Theory, Ed. Wiley Interscience

[12] R. Liu, L. Dow, E. Liu, "A survey of PEV impacts on electricity utilities", in IEEE PES Innovative Smart Grid Technologies Conf., 2011, pp. $1-8$

[13] G. Preetham, W. Shireen, "Photovoltaic station for plug-in hybrid electric vehicles in a smart grid environment", in IEEE PES Innovative Smart Gird Technologies Conf., 2012, pp. $1-8$.

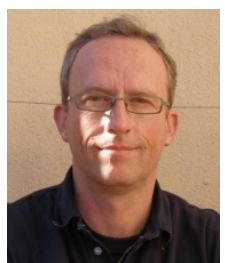

\section{BIOGRAPHY}

Ignasi Cairó (M’88) was born in Terrassa (Barcelona), Spain in 1967. He received the electronics and telecommunication degrees by Politechnical Universisty of Catalonia, Barcelona, Spain in 1988 and 1992 respectively.

He joined the R\&D dept. from Circutor in 1995 , involved in industrial power electronics and RFID systems. From 1999 until 2003 at Philips, and until 2008 at Epson Electronics $\mathrm{GmbH}$ he has been engaged in activities related to MMIC and RFIC design for wireless transceiver communications systems. From 2008 until now has been working in energy efficient systems in the industry as a innovation director for Circutor and now as a project leader of the area of Electricity and Power Electronics at IREC. Main research topics cover electronics in power converters, energy distribution systems, microgrids, and communications for the Smart grids.

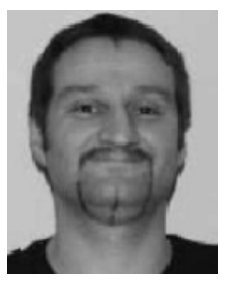

Andreas Sumper (S’05-M'08) was born in Villach, Austria. He received the

Dipl.-Ing in Electrical Engineering from the Technical University of Graz,

Graz, Austria, in 2000, and the Ph.D. degree in Electrical Engineering from

the Polytechnic University of Catalonia (UPC), Barcelona, Spain, in 2008.

From 2001 to 2002 he was a Project Manager for innovation projects in private industry. In 2002 he joined the Centre for Technological Innovation in Static Converters and Drives (CITCEA-UPC). Since 2006 he has been an Assistant Professor in the Department of Electrical Engineering, UPC. In CITCEA-UPC, he was engaged in technology transfer with local industry in the field of power quality, renewable energies and power systems. In 2009 he joined the Power Electronics and Electrical Engineering Group in the Catalonia Institute for Energy Research (IREC). His current research interests include power quality, electrical machines, power system studies and distributed generation. 